\title{
ESTUDIO FITOQUÍMICO CUALITATIVO PRELIMINAR Y CUANTIFICACIÓN DE FLAVONOIDES Y TANINOS DEL EXTRACTO ETANÓLICO DE HOJAS DE Desmodium vargasianum Schubert
}

\author{
Diana R. Rengifo Zevallos*
}

\begin{abstract}
RESUMEN
En este trabajo se investigó los principales metabolitos secundarios presentes en el extracto etanólico de hojas de Desmodium vargasianum Schubert "pie de perro". El tamizaje fitoquímico realizado en el extracto etanólico sugiere la presencia de compuestos fenólicos (flavonoides, taninos) y alcaloides. Se realizó la cromatografía en capa fina, dando positivo flavonoides, taninos y alcaloides. Finalmente se cuantificaron flavonoides totales y taninos totales. En la cuantificación de flavonoides totales por espectrofotometría UV-VIS se obtuvo $202,05 \pm 1,10 \mathrm{mg}$ QE/g de extracto etanólico (EE). En cuanto a la cuantificación de taninos totales por espectrofotometría UV-VIS se obtuvo 178,50 \pm 0,75 mg ATE/g de EE.
\end{abstract}

Palabras clave: Desmodium vargasianum Schubert, polifenólicos, flavonoides, taninos y alcaloides.

\section{PRELIMINARY QUALITATIVE FITOCHEMICAL STUDY AND QUANTIFICATION OF FLAVONOIDS AND TANNINS OF ETHANOLIC EXTRACT OF LEAVES OF Desmodium vargasianum Schubert}

\begin{abstract}
In this work the secondary metabolites of Desmodium. vargasianum Schubert "pie de perro" leaves ethanolic extract was investigated. The phytochemical screening realized on the ethanolic extract suggests the presence of phenolic compounds (flavonoids, tannins) and alkaloids. The thin layer chromatography was positive for flavonoids, tannins and alkaloids. Finally, total flavonoids and total tannins were quantified. In the quantification of total flavonoids by UV-VIS spectrophotometry, 202,05 $\pm 1,10 \mathrm{mg} \mathrm{QE} / \mathrm{g}$ of ethanolic extract (EE) was obtained. As for the total tannins quantification by UV-VIS spectrophotometry, $178,50 \pm 0,75 \mathrm{mg}$ TAE$/ \mathrm{g}$ EE was obtained.
\end{abstract}

Key words: Desmodium vargasianum Schubert, polyphenolics, flavonoids, tannins and alkaloids.

"Facultad de Farmacia y Bioquímica, Universidad Nacional Mayor de San Marcos, Jr. Puno 1002, Lima, Perú, soludimes@gmail.com 


\section{INTRODUCCIÓN}

La planta Desmodium vargasianum Schubert pertenece a la familia Fabácea, es una especie nativa que crece en Perú, Ecuador y Bolivia. En Perú crece en los departamentos de Ancash, Apurímac, Ayacucho, Cusco, Huancavelica, La Libertad, Lambayeque, Lima y Piura. Esta planta es un subarbusto conocida con los nombres comunes de "pie de perro" y "pega pega", es considerada como planta endémica ${ }^{1,2,3}$. El estudio etnobotánico de la planta, reportado en la literatura, hace referencia que las hojas se utilizan en decocción como calmante, diurética, contra enfermedades hepáticas, depurador del riñón y para dolores musculares, así como desinflamante ${ }^{2}$. En la provincia de Sullana, del departamento de Piura del Perú, esta planta es conocida como "miñate" y es de utilidad forrajera para el ganado ${ }^{4}$

Los flavonoides son metabolitos secundarios que se encuentran presentes en las plantas, los cuales tienen una estructura química de quince carbonos con dos anillos benceno unidos a través de un anillo pirano heterocíclico. Los flavonoides se clasifican de acuerdo a su estado de oxidación y patrón de sustitución del anillo pirano en flavonoles, flavonas, isoflavonas, flavononas, flavononoles y flavan-3-oles, mientras que los compuestos individuales dentro de una clase difieren en el patrón de sustitución de los anillos benceno. Los flavonoides son de especial interés en la investigación de productos naturales por presentar actividad biológica, tales como antioxidante, antiinflamatorio, hepatoprotector, antibacteriano y antiviral ${ }^{5}$.

Los alcaloides son compuestos nitrogenados, por lo general heterocíclicos básicos y con actividad biológica diversa. Las especies del género Desmodium pueden contener alcaloides tales como salsolina, salsolidina (tetrahidroisoquinolinas), derivados de $\beta$-fenetilaminas ${ }^{6}$ y derivados de triptamina ${ }^{7}$. En Desmodium gyrans fueron identificados 2 alcaloides, uno de estructura isoquinolínica (1-[(3,4-dietoxifenil) metil]-6,7-dietoxi-isoquinolina) y el otro pirazolinico (3,4-dimetil-4-nitro-2-pirazolina-5-ona $)^{8}$.

Los taninos son compuestos polifenólicos que se encuentran presentes como metabolitos secundarios en varias especies vegetales. Los taninos según su estructura química se clasifican en dos grupos: hidrolizables y condensados. Los taninos hidrolizables son ésteres de ácido gálico y ácido elágico glicosilados y los taninos condensados o proantocianidinas son polímeros de flavan-3-ol y/o flavan-3,4-diol. Los taninos según su estructura química presentan diferentes efectos farmacológicos tales como antioxidante y antimicrobiano, con actividad frente a Staphylococcus aureus, Streptococcus pneumoniae, Bacillus anthracis y Shigella dysenteriae y efecto cicatrizante 9 .

Los objetivos de la presente investigación fueron: 1) Determinar cualitativamente los principales metabolitos secundarios mediante el tamizaje fitoquímico y la cromatografía en capa fina del extracto etanólico de hojas de D. vargasianum Schubert 2) Cuantificar el contenido de flavonoides y taninos totales del extracto etanólico de hojas de $D$. vargasianum Schubert. 


\section{PARTE EXPERIMENTAL}

\section{Muestra}

La especie vegetal D. vargasianum Schubert, "pie de perro", fue recolectada en la provincia de Huarochirí del departamento de Lima, a 1100 msnm en el mes de agosto del 2015, durante la etapa de floración. La clasificación taxonómica de la planta fue realizada en el herbario del Museo de Historia Natural de la Universidad Nacional Mayor de San Marcos por el biólogo Severo Baldeón Malpartida.

La planta fue secada a la sombra a temperatura ambiente por dos semanas, se utilizó papel kraft, cambiándose de papel cada 24 horas. Se seleccionaron las hojas secas y fueron pulverizadas en un molino de cuchillas tipo Wiley. Se incorporó el polvo de hojas secas en frasco de vidrio estéril de color ámbar con tapa de polipropileno de color negro.

Se determinó la humedad de la planta mediante el método gravimétrico ${ }^{10}$. Se pesó $10 \mathrm{~g}$ de la muestra de hojas frescas en balanza analítica OHAUS y se secó hasta peso constante en estufa MEMMERT a $105^{\circ} \mathrm{C}$ durante 5 horas y se pesó la muestra. Se continuó con el secado y la pesada a intervalos de 1 hora hasta que la diferencia entre dos pesadas sucesivas no sea mayor de $0,25 \%$. De igual forma se procedió con las hojas secas, usándose una muestra de 2 gramos.

\section{Extracto etanólico}

Se incorporó $100 \mathrm{~g}$ de polvo de hojas secas de D. vargasianum Schubert en $1 \mathrm{~L}$ de etanol 70 y se maceró dentro del lapso de 24 horas de efectuada la molienda, en frasco ámbar de $2 \mathrm{~L}$ protegido de la luz y el calor y se agitó diariamente por 15 días. Luego, se filtró y se llevó a estufa de aire circulante a $40{ }^{\circ} \mathrm{C}$ en fuentes de vidrio de pirex. Se desecó en 4 días y se obtuvo un residuo de peso constante que se denominó extracto etanólico.

\section{Ensayo de solubilidad}

De manera secuencial se ordenó y etiquetó los tubos de ensayo con los nombres de los solventes de diferente polaridad ${ }^{11}$. Se introdujo a cada tubo de ensayo $20 \mathrm{mg}$ de extracto etanólico de hojas de D. Vargasianum Schubert y en cada tubo se añadió $1 \mathrm{~mL}$ de solvente (agua destilada, metanol, etanol $96 \%$, etanol $70 \%$, diclorometano, cloroformo, $\mathrm{n}$ hexano y éter dietílico). Se agitó cada tubo de ensayo y se procedió a observar los resultados de solubilidad por un tiempo máximo de 10 minutos.

\section{Tamizaje fitoquímico}

Se utilizó el extracto etanólico seco de hojas de D. Vargasianum Schubert para realizar las pruebas fisicoquímicas de caracterización, de cambios de coloración y formación de $\operatorname{precipitados}^{12}$.

\section{Cromatografía en capa fina}

Se realizó la cromatografía en capa fina, usando como fase estacionaria silica gel $60 \mathrm{GF}_{254} \mathrm{y}$ como fase móvil los solventes n-butanol: ácido acético : agua (BAW) (4:1:5) para confirmar 
la presencia de los metabolitos secundarios ${ }^{11}$. Se aplicó la siembra del extracto etanólico seco, previamente reconstituido en etanol $70 \%$ usando capilares nuevos. Los reveladores usados fueron: tricloruro férrico al $1 \%$ para taninos, Dragendorff para alcaloides y tricloruro de aluminio para flavonoides. En el caso de los flavonoides se observó la placa cromatográfica a luz UV de $365 \mathrm{~nm}$ antes y después del revelado.

\section{Cuantificación de flavonoides totales}

Se cuantificaron los flavonoides totales presentes en el extracto etanólico seco por espectrofotometría a una longitud de onda de $415 \mathrm{~nm}$ por triplicado, según literatura ${ }^{13}$. Se preparó la curva de calibración, se pesaron 2,70 mg de quercetina de grado Q.P. y se disolvió con etanol $80 \%$ en cantidad suficiente para $10 \mathrm{~mL}$ de solución. Luego se tomó sucesivamente $100 \mu \mathrm{L}, 175 \mu \mathrm{L}, 350 \mu \mathrm{L}$ y $750 \mu \mathrm{L}$ de la solución preparada previamente para diluirlo con etanol $80 \%$ hasta su enrase respectivo en fiolas $10 \mathrm{~mL}$ x 4, siendo las concentraciones teóricas de las soluciones estándares de 2,70 $\mu \mathrm{g} / \mathrm{mL}, 4,73 \mu \mathrm{g} / \mathrm{mL}, 9,45 \mu \mathrm{g} / \mathrm{mL}$ y $18,90 \mu \mathrm{g} / \mathrm{mL}$, respectivamente. A cada disolución preparada se añadió $200 \mu \mathrm{L}$ de acetato de sodio $1 \mathrm{M}$ y $200 \mu \mathrm{L}$ de nitrato de aluminio al $10 \%$, se dejó reposar por 40 minutos y se realizó la lectura en el espectrofotómetro UV-VIS a $415 \mathrm{~nm}$. Por otro lado, para la preparación de la muestra por triplicado se pesaron $30,9 \mathrm{mg}$ del extracto etanólico seco y se diluyeron en tres fiolas de $25 \mathrm{~mL}$ con etanol al $80 \%$. Se tomaron $0,5 \mathrm{~mL}$ de cada muestra y se diluyeron a $25 \mathrm{~mL}$. Se tomó una alicuota de $1 \mathrm{~mL}$ de cada fiola y se agregaron $200 \mu \mathrm{L}$ de acetato de sodio $1 \mathrm{M}$ y 200 $\mu \mathrm{L}$ de solución de nitrato de aluminio al $10 \%$. Se dejó reposar por 40 minutos. Finalmente, se procedió a leer las absorbancias de las muestras en el espectrofotómetro UV-VIS a $415 \mathrm{~nm}$ de longitud de onda. La cantidad de flavonoides fue expresada como mg quercetina equivalente por gramo de muestra.

\section{Cuantificación de taninos totales}

Se cuantificaron los taninos totales del extracto etanólico seco por espectrofotometría UV-VIS a $700 \mathrm{~nm}$ por triplicado, según literatura ${ }^{14}$. Se preparó previamente una curva de calibración a las concentraciones de 2, 4, 8, 10 y $25 \mathrm{ppm}$ del patrón ácido tánico. Se pesó 0,50 g de extracto etanólico seco y se disolvió en $50 \mathrm{~mL}$ de metanol - agua 25:25 v/v. El método consta de dos etapas. En la primera etapa se realizó varias diluciones de la muestra, se adicionó el reactivo de Folin-Ciocalteu y carbonato de sodio al $20 \%$ y se formó un complejo coloreado azul; asimismo, se preparó el blanco, sin la incorporación de la muestra. En la segunda etapa se realizó la separación de taninos usándose gelatina al $25 \%$, caolín 2,5\% y solución ácida de cloruro de sodio. Se agitó y filtró al vacío, posteriormente se adicionó el reactivo de Folin-Ciocalteu, solución de carbonato de sodio al $20 \%$ y agua desionizada; asimismo, se preparó el blanco, sin la incorporación de la muestra. Se tapó con papel de aluminio y se dejó reposar por 1 hora para la lectura en el espectrofotómetro. Finalmente, se procedió a leer las absorbancias del patrón ácido tánico para elaborar la curva de calibración, luego se realizó la lectura de la muestra de la primera etapa, la cual representa el contenido total de fenoles y la lectura de la muestra de la segunda etapa, que representa el contenido de los fenoles residuales. La cuantificación de los taninos totales se obtiene mediante la resta de las absorbancias de los fenoles totales y los fenoles residuales. El resultado fue expresado como $\mathrm{mg}$ de taninos equivalentes de ácido tánico por gramo de muestra. 


\section{RESULTADOS Y DISCUSIÓN}

\section{Determinación del porcentaje de humedad}

El porcentaje de humedad encontrado en las hojas frescas es de 72,90\% y en las hojas secas es de $7,53 \%$, como se presenta en la tabla 1 .

Tabla 1. Porcentaje de humedad de hojas frescas y secas de Desmodium vargasianum

\begin{tabular}{|c|c|c|c|c|}
\hline \multicolumn{5}{|c|}{ Muestra: Hojas frescas } \\
\hline \multirow[t]{2}{*}{$\mathrm{N}$} & Pesafiltro & Muestra & Pesafiltro + & \% Humedad \\
\hline & vacío (g) & humedad (g) & Muestra seca & \\
\hline 1 & 24,7752 & 10,0215 & 32,0473 & $72,54 \%$ \\
\hline \multirow[t]{2}{*}{2} & 24,7752 & 10,0215 & 32,0157 & $73,25 \%$ \\
\hline & & & & Media $=72,90 \%$ \\
\hline \multicolumn{5}{|c|}{ Muestra: Hojas secas } \\
\hline 1 & 23,1054 & 2,0324 & 23,2597 & $7,59 \%$ \\
\hline \multirow[t]{2}{*}{2} & 23,1054 & 2,0324 & 23,2572 & $7,47 \%$ \\
\hline & & & & Media $=7,53 \%$ \\
\hline
\end{tabular}

\section{Ensayo de solubilidad}

Con el ensayo de solubilidad se evidenció que el extracto etanólico de hojas de D. vargasianum Schubert es soluble en solventes polares, se presenta en la tabla 2.

\section{Tamizaje fitoquímico}

El método de tamizaje fitoquímico sugiere la presencia de determinados metabolitos secundarios en el extracto etanólico de las hojas de D. vargasianum (tabla 3).

Tabla 2. Ensayo de solubilidad del extracto etanólico de hojas de D. vargasianum

\begin{tabular}{lc}
\hline SOLVENTE & RESULTADO \\
\hline Agua destilada & ++ \\
Metanol & +++ \\
Etanol $96 \%$ & +++ \\
Etanol 70\% & +++ \\
Diclorometano & ++ \\
Cloroformo & - \\
n-hexano & - \\
Éter dietílico & - \\
\hline
\end{tabular}

Leyenda: -: Insoluble, +: Poco soluble, ++: Soluble, +++: Muy soluble 
Tabla 3. Tamizaje fitoquímico del extracto etanólico de hojas de D. vargasianum

\begin{tabular}{llc}
\hline \multicolumn{1}{c}{ METABOLITO } & REACCIÓN & RESULTADO \\
\hline Carbohidratos & Molish & + \\
Aminoácidos libres y & Antrona & + \\
grupos aminos & Ninhidrina & - \\
Compuestos fenólicos & Tricloruro férrico & ++ \\
Taninos & Gelatina-NaCl & + \\
Flavonoides & Shinoda & ++ \\
Triterpenoides y & Lieberman- Buchard & + \\
esteroides & & \\
Naftaquinonas, & Borntranger & - \\
antraquinonas y & & \\
antronas & & - \\
Antocianinas & Rosenheim & ++ \\
Alcaloides & Mayer & ++ \\
Saponinas & Dragendorff & ++ \\
Glucósidos & Wagner & - \\
\hline
\end{tabular}

Leyenda: -: Ausencia, +: Leve, ++: Moderado +++: Abundante

\section{Cromatografía en capa fina}

La cromatografía en capa fina confirmó la presencia de flavonoides, taninos y alcaloides en el extracto etanólico de hojas de D. vargasianum Schubert.

\section{Cuantificación de flavonoides}

La cantidad de flavonoides totales están expresados en $\mathrm{mg}$ de quercetina equivalente/g. Se obtuvo 202,05 $\pm 1,10 \mathrm{mg}$ QE/g de extracto etanólico de hojas de $D$. vargasianum, la ecuación de la curva de calibración del quercetina patrón fue: $\mathrm{Y}=0,0399 \mathrm{X}+0,0574, \mathrm{r}^{2}=0,9998$ con $p<5 \%$. En relación a la cantidad de flavonoides en plantas del género Desmodium se tiene el reporte que presenta diferencias cuantitativas significativas, así tenemos que $D$. gyran ${ }^{15}$ y D. gangeticum ${ }^{16}$ se halló que contienen 70,50 $\pm 2,00 \mathrm{mg} Q \mathrm{QE} / \mathrm{g}$ y 37,02 $\pm 0,15 \mathrm{mg} \mathrm{QE} / \mathrm{g}$ de extracto metanólico de hojas, respectivamente.

\section{Cuantificación de taninos}

La cantidad de taninos totales están expresados en $\mathrm{mg}$ de ácido tánico equivalente/g. Se halló 178,50 \pm 0,75 mg ATE/g de extracto etanólico de hojas de D. vargasianum, la ecuación de la curva de calibración del ácido tánico obtenida fue: $\mathrm{Y}=0,0952 \mathrm{X}+0,0493, \mathrm{r}^{2}=0,9996$ con $\mathrm{p}<5 \%$, esto representa a 1,80 $\pm 0,01 \mathrm{~g}$ ATE/100 g de hoja seca. La concentración de taninos es muy variable, dependiendo de la especie, tipo de tejido, cultivo, estado de desarrollo y condiciones ambientales. El contenido de taninos totales de especies forrajeras, principalmente de la familia Fabaceae, oscilan entre 1,57 a 9,16 g ATE/100 g de muestra seca $^{17,18}$. El contenido de 2 a $5 \%$ de taninos expresado como ácido tánico equivalente se consideran de moderada concentración, a partir de $6 \%$ se considera de alta concentración, en Desmodium molliculum se halló 5,17 $\pm 0,10 \mathrm{~g}$ ATE/100 g de planta seca ${ }^{19}$. 


\section{CONCLUSIONES}

1. En el tamizaje fitoquímico del extracto etanólico de hojas de D. vargasianum Schubert se encontraron compuestos fenólicos (flavonoides, taninos) como componentes mayoritarios, también se observó presencia alcaloides. La presencia de estos metabolitos secundarios fue confirmada mediante cromatografía en capa fina utilizando los reveladores específicos.

2. Se encontró que el extracto etanólico de hojas de D. vargasianum Schubert tiene un contenido de flavonoides de 202,05 $\pm 1,10 \mathrm{mg}$ QE/g y un contenido de taninos de $178,50 \pm 0,75 \mathrm{mg}$ ATE/g.

\section{AGRADECIMIENTO}

Agradecimiento al Instituto de Investigación de Ciencias Farmacéuticas y Recursos Naturales "Juan de Dios Guevara" y al Laboratorio de Farmacognosia y Medicina Tradicional de la Facultad de Farmacia y Bioquímica de la UNMSM por el apoyo brindado durante la realización de esta investigación. Mi agradecimiento al Dr. Pablo Enrique Bonilla Rivera, al Dr. César Máximo Fuertes Ruitón y mi agradecimiento póstumo a la Q.F. Bertha Jurado Teixeira.

\section{REFERENCIAS BIBLIOGRÁFICAS}

1. Baldeón S, Flores M, Roque J. Fabaceae endémicas del Perú en El libro rojo de las plantas endémicas del Perú. Rev Perú Biol. 2006; 13(2): 302s - 337s.

2. Mostacero J. Características edafoclimáticas y fitogeográficas de las plantas medicinales del dominio andino noroccidental del Perú, durante 1976 al 2004. [Tesis para optar el grado de doctor en medio ambiente]. Trujillo: Universidad Nacional de Trujillo; 2005. $313 \mathrm{p}$.

3. Cerna LA. Ciencia y tecnología de malezas. 1a ed. Trujillo: Fondo Editorial de la Universidad Privada Antenor Orrego; 2013.

4. Etnobotánica de las especies del monte ribereño en el río Chira, Sullana. [Tesis para optar el título profesional de Biólogo]. Piura: Universidad Nacional de Piura; 2015. 126 p.

5. Kumar S, Pandey AK. Chemistry and Biological Activities of Flavonoids: An Overview. The Sci World J. 2013; 2013:1-16.

6. Phillipson JD, Roberts MF, Zent MH. The chemistry and biology of isoquinoline alkaloids. 1a ed. Berlin: Springer Verlag; 1985.

7. Ganjhu RK, Mudgal PP, Arunkumar G. Pharmacological and Phytoconstituent Profile of Desmodium Gangeticum-An Update. IJPPR. 2014; 6(3): 643-657.

8. Gopalakrishnan S, Rajameena R. GC-MS. Analysis of some bioactive constituents of the leaves of Desmodium gyrans DC. IRJP. 2012; 3 (8): 271-274. 
9. Monteiro JM, Albuquerque UP, Araújo EL. Taninos: uma abordagem da química à ecologia. Química Nova. 2005; 28 (5): 892-896.

10. Farmacopea de los Estados Unidos 40 - Formulario Nacional 35 (USP 40 - NF 35). (561) Artículos de Origen Botánico. <921> Determinación de Agua. 2017; 1:458-459, $465,927$.

11. Lock de Ugaz O. Investigación fitoquímica. Métodos en el estudio de productos naturales. 2a ed. Lima: Fondo Editorial Pontificia Universidad Católica del Perú; 1994.

12. Gorriti A, Córdova A, Jurado B, Ortega EC, Retuerto MG. Manual de Laboratorio de Farmacognosia I. Lima: Cátedra de Farmacognosia y Medicina Tradicional, UNMSM. 2013.

13. Lock O, Cabello I, Doroteo V. Análisis de flavonoides en plantas. Pontificia Universidad Católica del Perú. [online]. 2006 [accesada el 01 de diciembre del 2006]. Disponible en: http://old.iupac.org/publications/cd/medicinal_chemistry/.

14. Lastra H, Rodríguez E, Ponce de León H, González ML. Método analítico para la cuantificación de taninos en el extracto acuoso de romerillo. Rev Cubana Plant Med. 2000; 5(1):17-22.

15. Vipin PS, Johannah NM, Menon S, Lincy L, Raghavamenon AC, Padikkala J. Antithrombotic and anticoagulant activities of Desmodium gyrans (DC). J Chem Pharm Res. 2015; 7(5):973-980.

16. Vaghasiya Y, Dave R, Chanda S. Phytochemical analysis of some medicinal plants from Western Region of India. Res J Med Plant. 2011; 5(5): 567-576.

17. Yusiati L, Kurniawati A, Hanim C, Anas M. Protein Binding Capacity of Different Forages Tannin. IOP Conf. Series: Earth and Environmental Science. 2018. doi :10.1088/1755-1315/119/1/012007

18. Alam M, Amin M, Kabir A, Moniruzzaman M y McNeill. Effect of Tannins in Acacia nilotica, Albizia procera and Sesbania acculeata Foliage Determined In vitro, In sacco, and In vivo. Asian-Aust J Anim Sci. 2007; 20(2): 220-228.

19. Gutiérrez M, Ortiz D, Muñoz G, Bah M y Serrano V. Contenido de sustancias antinutricionales de malezas usadas como forraje. Rev Latinoam Quím. 2010; 38(1): 58-67. 